\title{
Non-destructive Evaluation of Pore Size and Structural Change in Geopolymer Composite Materials with Added Fibres
}

\author{
Marcela Kolínová ${ }^{1}$, Totka Bakalova ${ }^{2}$, Lukáš Voleský1, Pavel Kejzlar², Vladimir Kovačíč ${ }^{2}$ \\ ${ }^{1}$ Institute for Nanomaterials, Advanced Technologies and Innovation, Technical University of Liberec, Studentska 2, 461 \\ 17 Liberec, Czech Republic, E-mail: marcela.kolinova@tul.cz, lukas.volesky@tul.cz \\ ${ }^{2}$ Faculty of Mechanical Engineering, Department of Material Science, Technical University of Liberec, Studentska 2, 461 \\ 17 Liberec, Czech Republic, E-mail: totka.bakalova@tul.cz, pavel.kejzlar@tul.cz, vladimir.kovacic@tul.cz
}

\begin{abstract}
This paper introduces a non-destructive method of analysis of the internal structure, occurrence and pore size in a specific volume of geopolymer composite material, with and without the addition of inorganic fibres in the form of meshes using computed tomography. A desktop micro-tomography device Skyscan 1272 was used for visualization and accurate analysis of the interlacing points in the basalt and carbon fibre meshes and for evaluating the adhesion of the fibres to the matrix of the composite material. An appropriate resolution was selected both for displaying the interlacing points in the inorganic fibres and for visualization and analysis of the geopolymer structures. The method makes it possible to evaluate the differences in the structure of the studied materials in a non-destructive way and to check the adhesion of the fibres to the base material.
\end{abstract}

Keywords: Geopolymer composite, Inorganic fibres, Interlacing point, Adhesion, Computed tomography

\section{Introduction}

$\mathrm{X}$-ray computed tomography is used to characterize the composite microstructure and porosity of a material. This method can produce non-destructive and three-dimensional (3D) images to quantify properties of the micro-structure such as pore size distribution, porosity and tortuosity of the porous network. X-ray radiography physics is based on the Beer-Lambert law [1, 2 and 3]. Pores are distinguished in X-ray computed tomography on the basis of their linear attenuation coefficient, $\mu$. This parameter depends on the electron density of the sample, the effective atomic number of the sample, and the energy of the incoming X-ray beam. The method comprises an $\mathrm{X}$-ray source, a rotation state on which the object is fixed, an X-ray detector and reconstruction software [1]. Construction of a 3D map of a sample involves the recording of several X-ray radiographs of the sample at different viewing angles. This imaging information is then used in reconstruction software to recalculate the 3D map of the attenuation from the combination of the obtained radiographs.

The crucial point in applying tomography to materials science is the achievable spatial resolution. For a limit resolution in the order of $8 \mu \mathrm{m}$, a cone-beam system can be used with a conventional micro-focus X-ray tube as the source. Most recent laboratory systems routinely achieve resolutions as low as $5 \mu \mathrm{m}$. Larger samples have a lower resolution, limited by the number of pixels. The best quality images in terms of signal-to-noise ratio and spatial resolution allowing high-resolution micro-tomography are obtained on synchrotron radiation [4].

There are several disadvantages in the use of X-ray computed tomography porosity analysis. 3D methods are not always applicable if samples are too large or small; they are very expensive and cannot distinguish touching grains of the same mineral nor can they clearly separate different minerals with very similar properties. $[5,6]$

This article illustrates the possibilities of using X-ray tomography as a non-destructive method for material evaluation. This method allows non-destructive testing of the internal structure of a composite material, evaluation of the porosity of the structure and allows for the adhesion of added fibres to the matrix of a geopolymer composite.

\section{Experimental Procedure}

Non-destructive imaging methods such as X-ray computed tomography (CT) yield high-resolution, three-dimensional representations of pore space and fluid distribution within a porous material. Investigation of the hydro-mechanical characteristics of geomaterials has still many limitations. In particular, a reliable estimation of permeability is a matter of the utmost importance in many fields where reliable barriers are required to prevent flow of radioactive contaminants, oil and gas from underground storages. In this respect, engineers dealing with very fine soil materials like clay have been searching for more advanced techniques for determining porosity, pore-size distribution and permeability under various field conditions. Visualizing and quantifying micro-scale internal structures and the physical composition of a material in an undisturbed state is now feasible using 3D images by X-ray CT methodology.

A CT-Analyser (CTAn) is a device for measuring quantitative parameters and constructing visual models from scanned 3D datasets obtained with SkyScan micro CT instruments. CTAn performs quantitative measurements of both densitometry (voxel coefficient of calibrated density) and morphometry. Morphometry image analysis is based on binary images, which contain only black and white pixels, representing the non-selected or selected pixels, respectively. In CTAn, the binarization of images is performed by thresholding, whereby segmented images are the result of this process and simple global or adaptive methods allow for easy segmentation.

Porosity analysis is a morphometric analysis, this image analysis is performed by CTAn on selected pixels, i.e. 
white pixels, better known as an "object". Within this object, CTAn can identify pores as being a number of black pixels surrounded by white pixels.

The effect of introducing additives to the basic matrix of the geopolymer composite on the resulting porosity and particle size distribution was studied. Sand and basalt fibres were added as an additive to the geopolymer matrix. Mesh-shaped fibres have a substantial effect on the improvement of the mechanical properties of the geopolymer composite. Therefore, in the second part of the study, the effect of the added mesh-shaped fibres on the resulting porosity in the specifically examined volume of the composite was observed and the adhesion of the fibres to the base material was monitored. The important points in the geopolymer composite are the so-called interlacing points where the adhesion of fibres to the base material is decisive for the concentration of air bubbles.

At the beginning of the experiment, three types of samples were prepared, i.e. a geopolymer composite (Sample-G), a geopolymer composite with sand (SampleGS), and a geopolymer composite with fibres (SampleGF). The samples were prepared according to the requirements of the non-destructive method used and with dimensions suitable for scanning (Table 1).

\section{Tab. 1 Description and dimensions of tested samples}

\begin{tabular}{|c|c|c|}
\hline Labelling of the samples & Composition of the samples & Dimensions of the samples [mm] \\
\hline Sample-G & Geopolymer & $5.9 \times 6.6 \times 5.6$ \\
\hline Sample-GS & Geopolymer with sand & $7.7 \times 8.0 \times 6.3$ \\
\hline Sample-GF & Geopolymer with fibres & $6.9 \times 4.6 \times 5.8$ \\
\hline
\end{tabular}

\section{Results and Discussion}

The following parameters were selected for scanning the geopolymer structures: source voltage $100 \mathrm{kV}$; source current $100 \mu \mathrm{A}$; image pixel size $4.5 \mu \mathrm{m}$; exposure 3,330 $\mathrm{ms}$; use $\mathrm{Cu} 0.11$ filter, rotation step $0.2^{\circ}$ and scan duration $135 \mathrm{~min}$.

Figures 1 to 3 show the region of interest (in green areas, the figures on the right) selected for geopolymer structure analysis using the CTAn analysis software for the three composite materials labelled Sample-G, Sample-GS and Sample-GF. After scanning the samples using special software, the region of interest (Figures 1-3 on the left) was determined, which is the same for all of the samples, i.e. the volume of the examined area is $47.33 \mathrm{~mm}^{3}$. The porosity was evaluated in these selected regions and the results obtained along with the standard deviation are

described in Table 2. In Figures 1-3 on the left, the structure of the geopolymer composite materials is shown on the transaxial and sagittal planes. Figures 1-3 on the right show the change in the structure of the base matrix in the basic state (Figure 1 on the right), after adding the sand (Figure 2 on the right) or after adding the basalt fibres (Figure 3 on the right).

Tab. 2 Measured porosity of the examined samples

\begin{tabular}{|c|c|c|c|}
\hline & Porosity & & \\
\hline $\begin{array}{l}\text { Total VOI vo- } \\
\text { lume }\end{array}$ & $\begin{array}{l}\text { Sample- } \\
\text { G }\end{array}$ & $\begin{array}{l}\text { Sample- } \\
\text { GS }\end{array}$ & $\begin{array}{l}\text { Sample- } \\
\text { GF }\end{array}$ \\
\hline \multirow{3}{*}{$47.33 \mathrm{~mm}^{3}$} & 28.95 & 55.54 & 54.97 \\
\hline & 28.88 & 59.63 & 54.83 \\
\hline & 29.29 & 56.30 & 56.71 \\
\hline Average & 29.04 & 57.16 & 55.50 \\
\hline SD & 0.18 & 1.78 & 0.86 \\
\hline
\end{tabular}
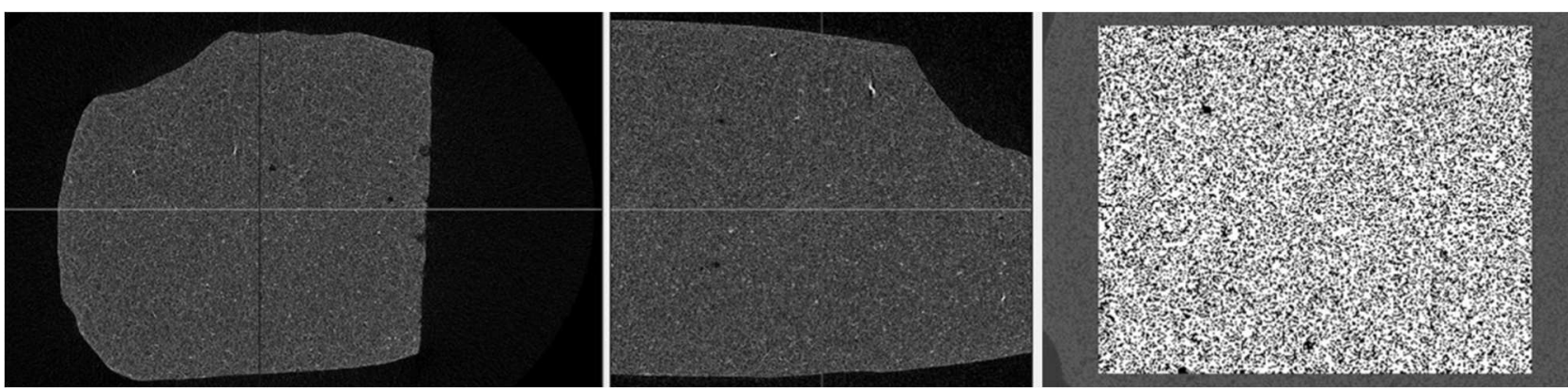

Fig. 1 Structure of the geopolymer composite material (Sample-G) on the transaxial and sagittal planes (on the left) and the region of interest $\left(47.33 \mathrm{~mm}^{3}\right.$ ) limited by the green field (on the right)
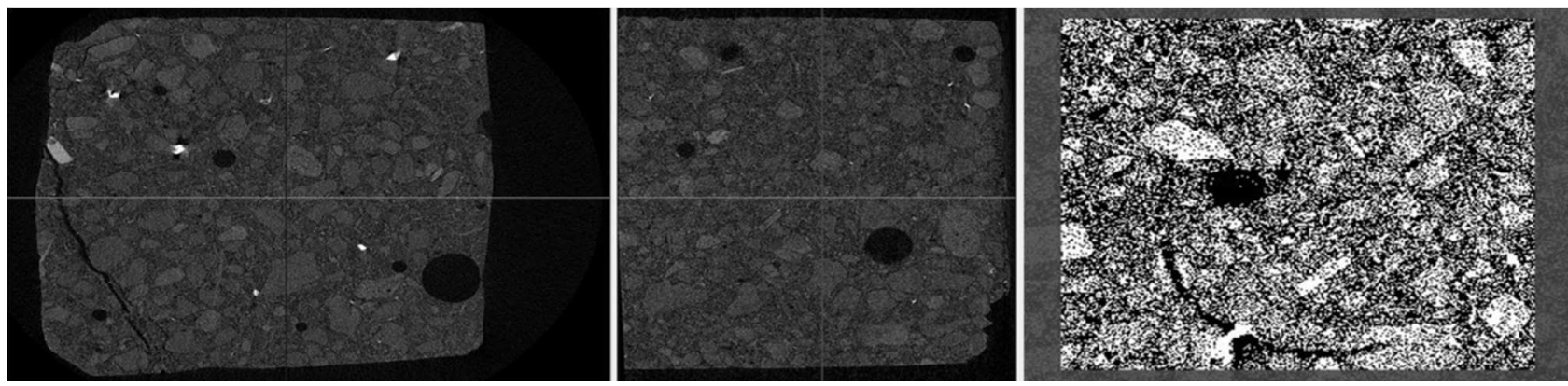

Fig. 2 Structure of the geopolymer composite material with sand (Sample-GS) on the transaxial and sagittal planes (on the left) and the region of interest $\left(47.33 \mathrm{~mm}^{3}\right)$ limited by the green field (on the right) 

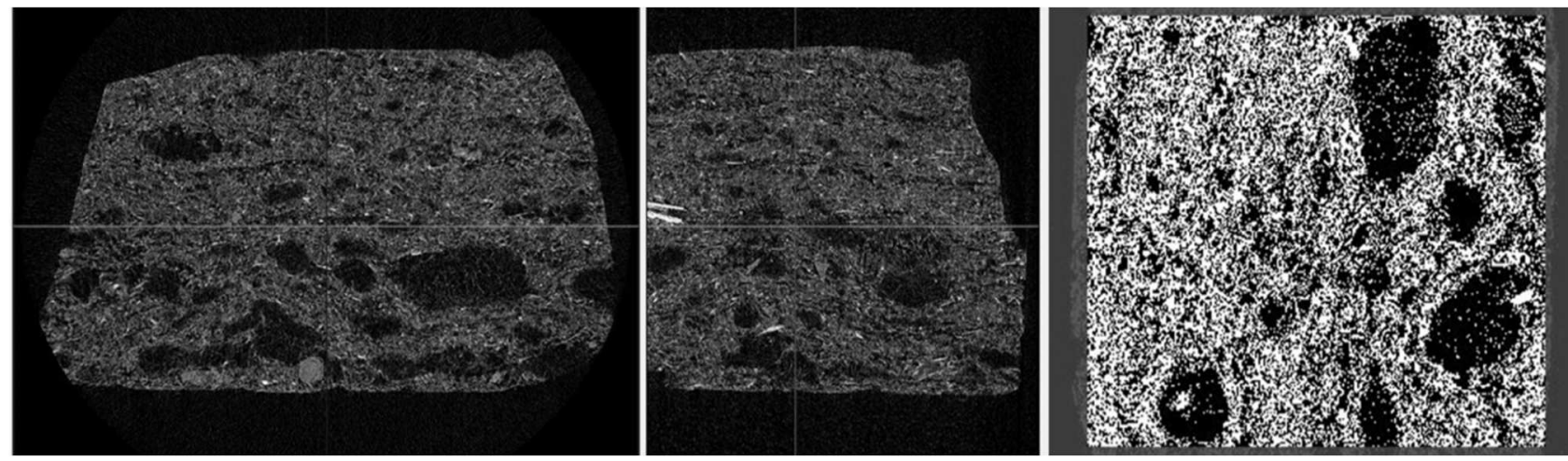

Fig. 3 The structure of geopolymer composite material with fibres (Sample-GF) on the transaxial and sagittal planes (on the left) and the region of interest $\left(47.33 \mathrm{~mm}^{3}\right.$ ) limited by the green field (on the right)

The X-ray computed tomography method used (see Table 2) shows that the percentage of porosity increases after the addition of sand or basalt fibres in the volume of composite material examined. The measured average porosity in the investigated composite volume $\left(47.33 \mathrm{~mm}^{3}\right)$ labelled Sample G is $29.04 \%$. After the addition of sand to the composite system, the average porosity increased by about $49 \%$ (the measured value is $57.16 \%$ ) and after addition of basalt fibres by about $48 \%$ (the measured value is $55.50 \%$ ).

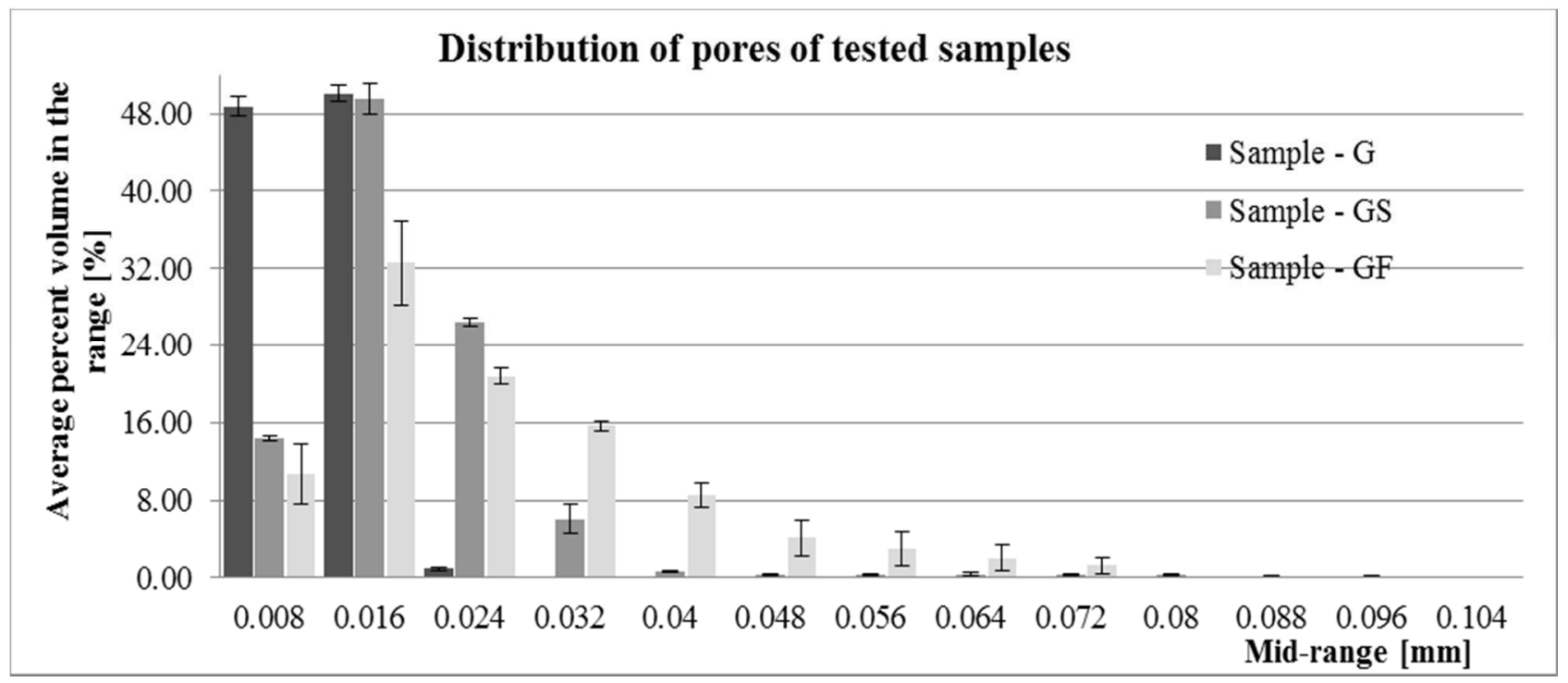

Fig. 4 Structure separation distribution of the tested samples, average percent volume in the range [\%]

The structure separation distribution and its occurrence in the matrix of the composites studied were measured three times and the results are described in Fig. 4. By analysing the sample of the geopolymer itself and the sand or fibre composites, the influence of the added materials (sand, fibres) on the porosity (see Table 2) and the distribution of the pores in the structures of the test samples (Fig. 4) were determined. The non-additive geopolymer contains about $50 \%$ of pores with a mean value of $0.009 \mathrm{~mm}$ to $0.018 \mathrm{~mm}$. The number of pores with a higher mean size is negligible. The sample composed of a sand-containing geopolymer is about $14 \%$ of the pore size of $0.009 \mathrm{~mm}, 50 \%$ of the pores with a mean value of $0.018 \mathrm{~mm}$ and approximately $26 \%$ of the pore size of $0.027 \mathrm{~mm}$. The number of larger pores is negligible. When fibres are added to the composite sample, approximately $10 \%$ of the pores are represented by a mean size of $0.009 \mathrm{~mm}$, approximately $32 \%$ of the pores a size of $0.018 \mathrm{~mm}, 20 \%$ of the pores by a size of $0.027 \mathrm{~mm}, 15 \%$ of the pores by a size of $36 \mu \mathrm{m}$ and approximately $8 \%$ of the pores by a size of $0.045 \mathrm{~mm}$.

In the network of inorganic fibres, the interlacing points were examined using X-ray computer tomography. The following parameters were set to scan these binding points: source voltage $80 \mathrm{kV}$; source current $125 \mu \mathrm{A}$; image pixel size $4.0 \mu \mathrm{m}$; exposure $1,954 \mathrm{~ms}$; use $\mathrm{Al} 1 \mathrm{~mm}$ filter, rotation step $0.2^{\circ}$ and scan duration $125 \mathrm{~min}$.

Figure 5 provides a preview (transaxial and sagittal view of the composite structure) of a binding point on the basalt fibre network and Figure 6 incorporates the interlacing point into the geopolymer composite. To scan the geopolymer composite with the network, the optimal scanning parameters were the same as for inorganic fibre interlacing points. 

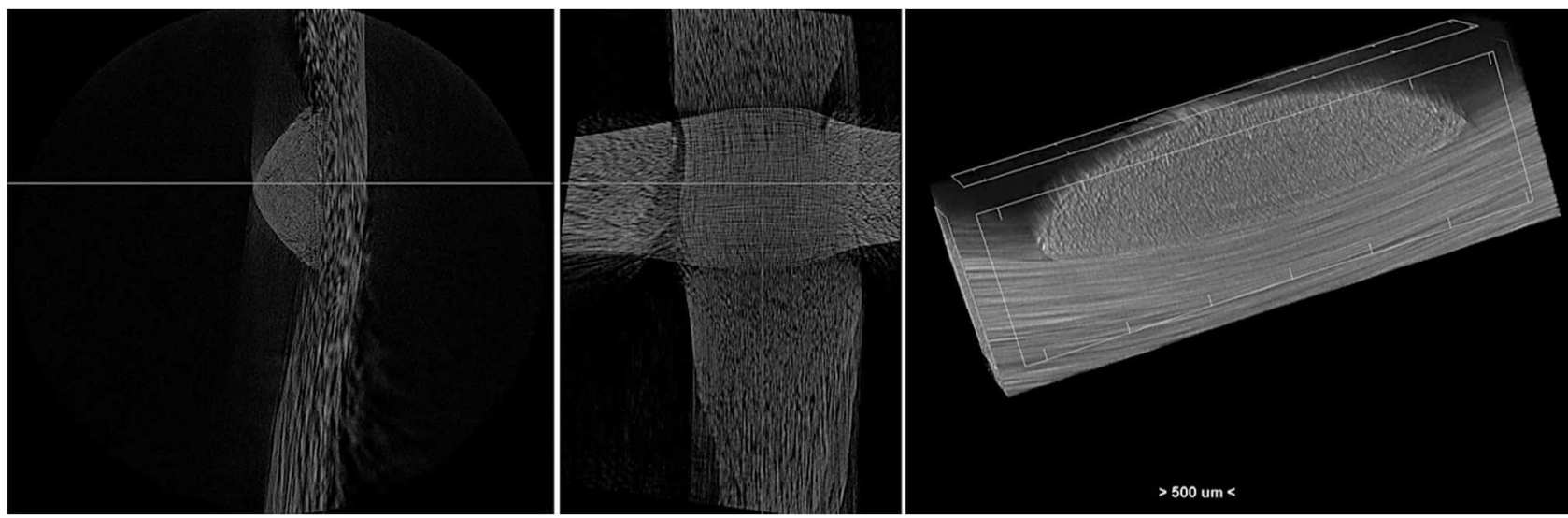

Fig. 53 visualization of the structure of inorganic fibre networks using DataViewer a) on the transaxial and sagittal planes and b) cut at an interlacing point
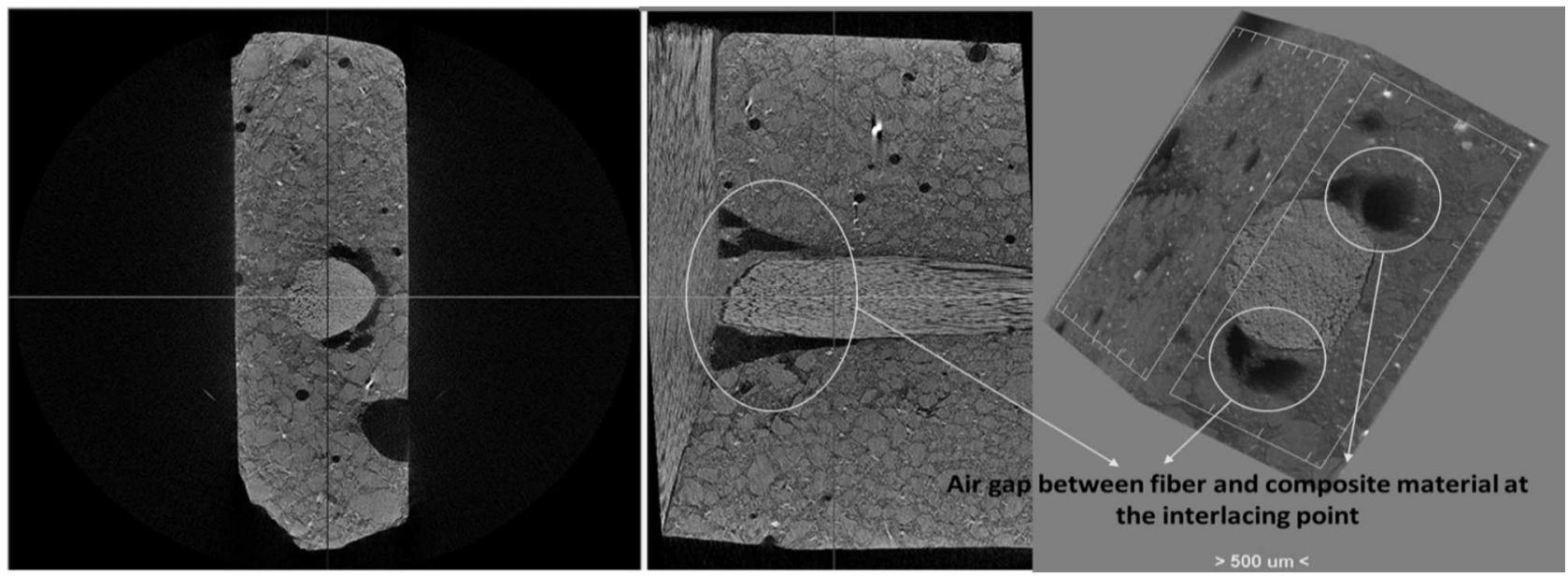

Fig. 6 Structure of the composite material with inorganic fibre additions on the transaxial and sagittal planes (on the left part of the figure) and various sections of the geopolymer fibre composite (on the right part of the figure)
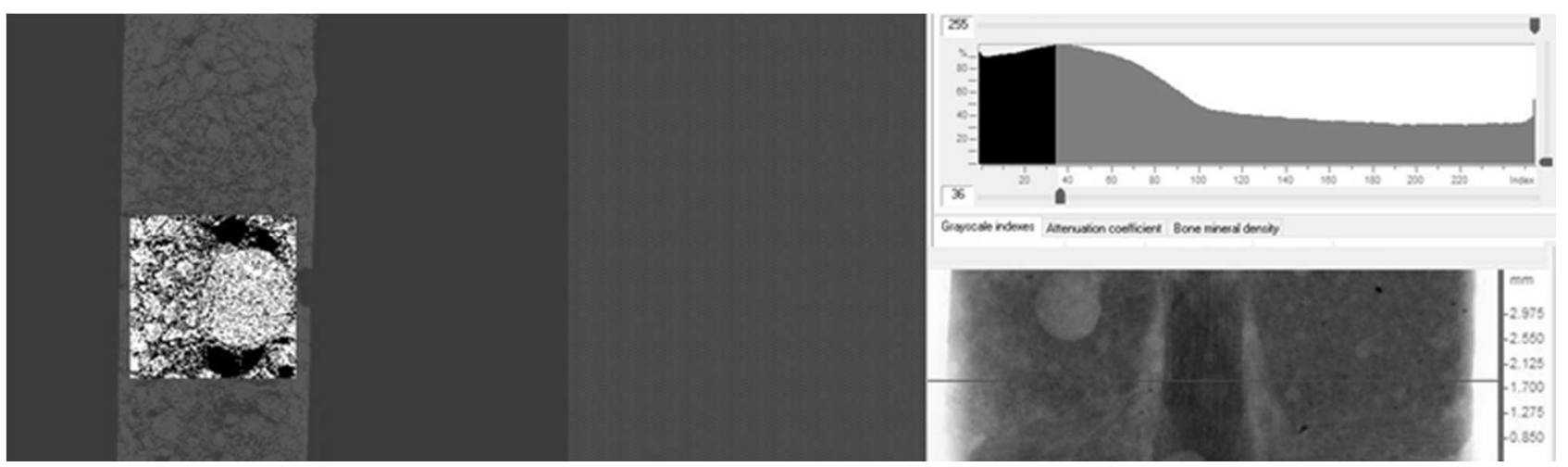

Fig. 7 Representation of the region of interest for geopolymer structure analysis with the addition of fibres using CTAn analysis software

Geopolymer composites doped with sand were selected as a basic composite in which the inorganic fibres in the form of networks are incorporated. Figure 6 shows the structure of the composite material on the transaxial and sagittal planes. It is quite clear that most of the pores in this material are centred on the boundary of the geopolymer and the network of inorganic fibres. The following Figure 8 shows that the composite structure has the largest pore size of $0.016 \mathrm{~mm}$ and the pores in the range of $0.004 \mathrm{~mm}$ to $0.084 \mathrm{~mm}$ are the percentage most represented. The number of pores larger than $0.084 \mathrm{~mm}$ in this structure is negligible.

Figure 7 shows the region of interest selected for geopolymer structure analysis using the CTAn analysis software for a composite material with a basalt fibre network. The porosity was evaluated in the selected area on the left of Figure 7 and the measured value with standard deviation is $79.95 \pm 0.16$. 


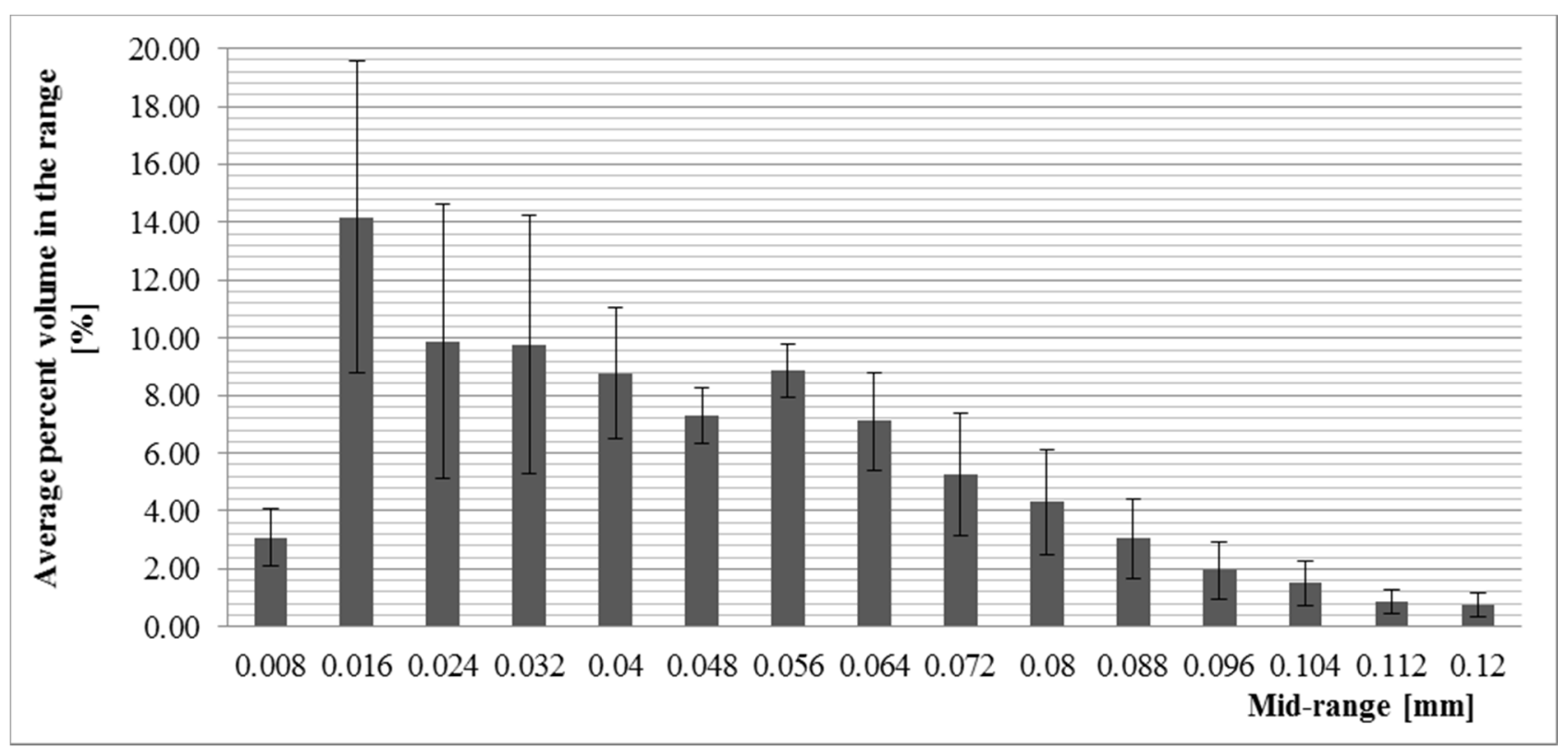

Fig. 8 Structure separation distribution in the composite material incorporating a basalt fibre network

Euler analysis provides a measure of connectivity density (Table 3), indicating the number of redundant connections between trabecular structures per unit volume. The Euler-Poincare number (Euler number) is an indicator of connectedness of a 3D complex structure. The Euler number is characteristic of a three-dimensional structure which is topologically invariant. It is a measure of how many connections in a structure can be severed before the structure falls into two separate pieces. The Euler-Poincare formula for a $3 \mathrm{D}$ object $\mathrm{X}$ is given by formula (1):

$$
\chi(X)=\beta_{0}-\beta_{1}+\beta_{2}
$$

Where $\beta_{0}$ is the number of objects; $\beta_{1}$ is the connectivity, and $\beta_{2}$ - the number of enclosed cavities.

Tab. 3 Euler numbers and connectivity obtained using specific analysis software

\begin{tabular}{|l|l|l|l|l|l|l|}
\hline Samples & \multicolumn{4}{|l|}{ Sample - G } & Sample - GS & \multicolumn{2}{l|}{ Sample - GF } \\
\hline Measurement & EN & C & EN & C & EN & C \\
\hline 1 & -1927508 & 2030430 & -2458437 & 2721104 & -1832052 & 2443101 \\
\hline 2 & -1923531 & 2026445 & -2451932 & 2715043 & -1882813 & 2403663 \\
\hline 3 & -1927652 & 2031724 & -2521260 & 2638039 & -1896472 & 2415552 \\
\hline Average & $\mathbf{- 1 9 2 6 2 3 0}$ & $\mathbf{2 0 2 9 5 3 3}$ & $\mathbf{- 2 4 7 7 2 1 0}$ & $\mathbf{2 6 9 1 3 9 5}$ & $\mathbf{- 1 8 7 0 4 4 6}$ & $\mathbf{2 4 2 0 7 7 2}$ \\
\hline SD & 1909 & 2247 & 31261 & 37810 & 27715 & 16518 \\
\hline
\end{tabular}

Remark: EN - Euler number; C-Connectivity.

Connectivity is related to the Euler number. When Euler number is lower, connectivity is higher and vice versa. This means that the sample contains a large amount of pores. According to the measurement results of Euler's number, it can be assumed that by adding additives (sand, fibres) to the geopolymer matrix the samples do not differ topologically probably.

Study [7] investigated the microstructural effects of hemp fibre and shiv addition on modified mortars by using X-ray tomography. The results showed that the porosity level in the hemp shiv modified mortar with regard to a weight content of 2 wt. \% was $5.08 \%$, whereas porosity in the case of hemp fibre modified mortar was $3.54 \%$.

Pores smaller than $1 \mathrm{~mm}^{2}$ account for between 8 and $30 \%$ of the pore area [8]. Literary source [9] studied the change in volumetric ratios of visible air voids related to a consolidation process on a macro scale using X-ray tomography. The results showed that the ratio of macro scale air voids to micro scale air voids changed significantly during the consolidation process.
Literary source [10] studied the porosity of coir fibres using X-ray tomography analysis. The porosity of the coir fibres using the X-ray tomography method ranged from 27 to $40 \%$. The fibre porosity analysed with this method was likely to have been overestimated because the method is based on the densitometry principle, and there is only a small difference in density between coir fibres and air. In addition, coir fibres consist of various thin organic tissues, which may not be detected on the scanned images.

Increasing the percentage of garnet in a geopolymer composite [6] increases the porosity from $0.15 \%$ (without adding garnet) to $2.89 \%$ (after adding $5 \%$ garnet) and to $10.95 \%$ (after adding $15 \%$ garnet).

After the addition of sand or basalt fibres to a geopolymer composite, the porosity in a pre-defined volume increased by approximately $50 \%$. Incorporation of basalt fibres in the form of networks to the geopolymer composite with the addition of sand resulted in the formation of air bubbles during the burning of fibres and the composite in 
the area of the interlacing points. The X-ray tomography analysis illustrates the surroundings of the interlacing points, and Figure 5 shows the concentrated air around the fibres. Reduced adhesion between the composite and the basalt fibres can be defined in these critical areas of the interlacing points.

\section{Conclusion}

In summary, X-ray tomography analysis is a good tool for studying the porosity and the internal structure of materials in general. It is a non-destructive method that reliably illustrates the occurrence of air bubbles in the investigated structure and provides information about their classification in the material volume. In order to supplement their quality, the results of this method should be compared with other additional measurements such as scanning electron microscopy. The composite material with the added sand and basalt fibre network shows an increase in porosity $(79.95 \pm 0.16)$ compared to the basic state of the composite material with sand $(57.16 \pm 1.78)$. This is due to the presence of air bubbles in the vicinity of the interlacing points and the associated poor adhesion between the fibres and the composite material. After evaluation of the Euler numbers and connectivity, it was shown that after the addition of the additives to the geopolymer matrix, changes in the topology of the individual samples were not observed.

\section{Acknowledgement}

The results of the project "Application of geopolymer composites as fire, $A G K$ ", registration number VI20172019055, were obtained through the financial support of the Ministry of Interior in the programme "The Safety Research of the Czech Republic" 20152020 (BV III/1-VS). We also acknowledge the financial support of the Ministry of Education, Youth and Sports for the project LO1201, performed in the framework of the targeted support of the "National Programme for Sustainability I" and of the Technical University of Liberec as part of the project "The study and evaluation of the material's structure and properties" with the support of the Specific University Research Grant, as provided by the Ministry of Education, Youth and Sports of the Czech Republic in the year 2018. We would also like to thank Craig Hampson for his help with English language correction.

\section{References}

[1] MAIRE, E., BUFFIERE, J. Y., SALVO, L., BLANDIN, J. J., LUDWIG, W., LETANG, J. M.
(2001). On the application of X-ray microtomography in the field of materials science. pp. 539546, Adv. Eng. Mater. 3.

[2] DOUGAL, A., JERRAM, HIGGINS, \& MICAHAEL, D. (2007). 3D analysis of rock textures: quantifying igneous microstructures. pp. 239-245, Elements 3.

[3] ROCHE, R. C., ABEL, R. A., JOHNSON, K. G., PERRY, C. T. (2010). Quantification of porosity in acropora pulchra (brook 1891) using X-ray micro-computed tomography techniques, pp. 1-9, J. Exp. Mar. Biol. Ecol. 396.

[4] MAIRE, E. (2012). X-ray tomography applied to the characterization of highly porous materials. pp. 136-178, Annu. Rev. Mater. Res. 42.

[5] BAKALOVA T. and M. KOLÍNOVÁ (2014). The possibility of using the Computerized Tomography in technique, No. 4, pp. $111-114$, ISSN 0447-6441, Fine mechanics and optics.

[6] BAKAlOVA, T., M. KOLÍNOVÁ and P. LOUDA (2014). Micro CT Analysis of Geopolymer Composites, Vol. 14, No. 4. pp. $505-510$. ISSN 1213-2489. Manufacturing technology.

[7] MOSTEFAI, N., HAMZAOUI, R., GUESSASMA, S., AMADOU, A.W., NOURI, H. (2015). Microstructure and mechanical performance of modified hemp fibre and shiv mortars: discovering the optimal formulation. pp. 359-371, Mater. Des. 84.

[8] KAIN, G., JOHANN, C.P., MARIUS-CATALIN, B., BERNHARD, P., KLAUS, R., ALEXANDER, P. (2015). Analyzing wood bark insulation board structure using X-ray computed tomography and modelling its thermal conductivity by means of finite difference method, pp. 112, J. Compos. Mater. 0(0).

[9] WILLIAMS, J., LAWRENCE, M., WALKER, P., (2015). Thermally modelling bio-composites with respect to an orientated internal structure. Sustainable ecological engineering design for society, Leeds, 17 th - 18th, September.

[10] TRAN, L.Q.N., MINH, T. NGUYEN, FUENTES, C.A., CHI, T., TRUONG, VUURE, VAN, A.W., VERPOEST, I. (2015). Investigation of microstructure and tensile properties of porous natural coir fibre for use in composite materials. pp. 437-445, Ind. Crops Prod. 65. 